\title{
A Distributed Dual-Band $L C$ Oscillator Based on Mode Switching
}

\author{
Guansheng Li, Student Member, IEEE, and Ehsan Afshari, Member, IEEE
}

\begin{abstract}
In this paper, we present a distributed dual-band $L C$ oscillator suitable for low-phase-noise applications. It switches between the odd and even resonant modes of a fourth-order $L C$ resonator. In contrast to other switched-resonator designs, the switches used for mode selection do not carry current, and therefore, do not affect the quality factor of the resonator, which leads to low phase noise. Analysis shows it achieves the same phase-noise figure-of-merit (FoM) as a single-band $L C$ oscillator that uses the same inductor and active core. This was verified by a prototype in a $0.13-\mu \mathrm{m}$ CMOS process. It draws a current of $4 \mathrm{~mA}$ from a 0.5-V power supply and achieves a FoM of $194.5 \mathrm{~dB}$ at the 4.9-GHz band and 193.0 $\mathrm{dB}$ at the 6.6-GHz band, which is the same as the reference standalone $L C$ oscillator. There is good agreement among theory, simulation, and measurement results.
\end{abstract}

Index Terms-Dual-band oscillator, high-order $L C$ resonator, low phase noise, low supply voltage.

\section{INTRODUCTION}

W IRELESS design is developing from single-mode to multimode systems that support multiple standards at several frequency bands. A major challenge for these systems is to design local oscillators (LOs) that cover the wide spectrum and meet the stringent phase-noise requirement. This is usually beyond the capability of a single-tank $L C$ voltage-controlled oscillator (VCO) using varactors for continuous frequency tuning. As a result, there has been an increasing interest in $L C$ oscillators that switch between multiple frequency bands [1]-[12].

One multiband scheme is to implement multiple $L C$ oscillators at different frequencies and enable one of them at a time [1]. However, this scheme can be improved, in the sense that there are always one or more inductors in idle. As will be explained later, these idle inductors could have been utilized to enhance the working LO's phase noise. Another technique is to use a switched resonator, in which the inductance and capacitance of the $L C$ resonator are controlled by MOS switches [2]-[4]. However, these switches usually insert resistance to critical current paths, which degrades the resonator's quality factor and deteriorates phase noise significantly [5], [6]. Several

Manuscript received May 28, 2010; revised October 15, 2010; accepted October 22, 2010. Date of publication December 03, 2010; date of current version January 12, 2011. This work was supported by the National Science Foundation (NSF) under NSF Grant DMS-0713732. The work of E. Afshari was supported by the NSF under Early Career Award ECCS-0954537.

The authors are with the School of Electrical and Computer Engineering, Cornell University, Ithaca, NY 14850 USA (e-mail: g1246@ cornell.edu; ehsan@ece.cornell.edu).

Digital Object Identifier 10.1109/TMTT.2010.2091203

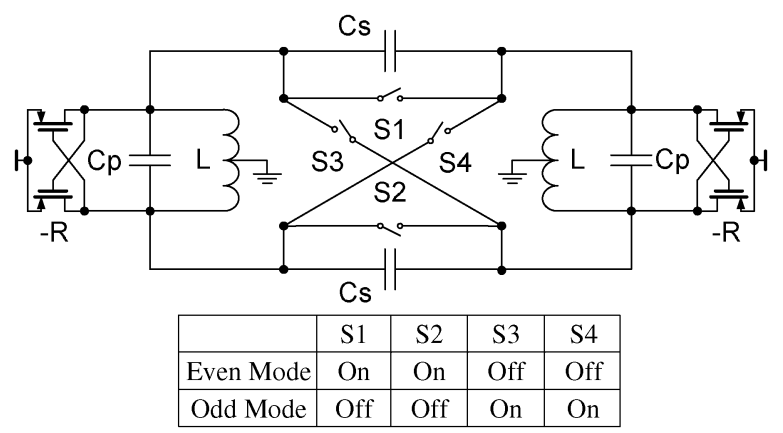

Fig. 1. Schematic of the proposed dual-band oscillator.

other structures were also proposed [7]-[11], and [12] summarized existing wideband/multiband VCO techniques. However, the phase-noise performance of these state-of-the-art multiband oscillators is generally inferior to single-band $L C$ oscillators [13], [14], which demonstrates the tradeoff between phase noise and frequency tuning range in oscillator design.

In this paper, we propose a dual-band $L C$ oscillator, which achieves band switching without impairing phase-noise performance. As shown in Fig. 1, two center-taped inductors and four capacitors comprise a differential resonator. It has an even and an odd resonate mode. Their frequencies are not harmonically related. A switching network is used to select one from the two resonant modes, and two p-type field-effect transistor (PFET) pairs are used to compensate the resonator's energy loss and sustain oscillation. Thus, one can get dual-band LO output from either PFET pair.

In contrast to other switched-resonator designs, there is no current going through the switches during steady oscillation because the switches that are turned on only damp the undesired mode. As a result, the working-mode quality factor of the resonator is not affected by the switches, and the oscillator can achieve low-phase noise. Analysis shows, this dual-band oscillator achieves the same phase-noise figure-of-merit (FoM) as a single-band $L C$ oscillator that uses the same inductor and active core (Fig. 2), where the FoM is defined as [15]

$$
\mathrm{FoM}=10 \log _{10}\left[\frac{1}{P_{\mathrm{diss} \mid \mathrm{mW}}}\left(\frac{f_{0}}{\Delta f}\right)^{2}\right]-L(\Delta f)
$$

in which $f_{0}$ is the center frequency, $\Delta f$ is the offset frequency, $L(\Delta f)$ is the phase noise in $\mathrm{dBc} / \mathrm{Hz}$, and $P_{\text {diss }} \mid \mathrm{mW}$ is the power consumption in $\mathrm{mW}$. This analysis was verified by a prototype in a $0.13-\mu \mathrm{m}$ CMOS process, in which the dual-band oscillator achieves $3-\mathrm{dBc} / \mathrm{Hz}$ lower phase noise while consuming 3-dB 


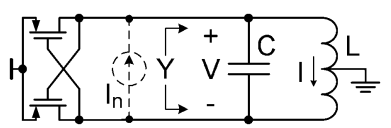

Fig. 2. Schematic of a single-band $L C$ oscillator.

more power, compared to a reference single-band $L C$ oscillator using the same inductor and active core.

Compared to the two-oscillator scheme, the proposed dual-band oscillator can be considered as two oscillators coupled through capacitors, which readily achieves $3-\mathrm{dBc} / \mathrm{Hz}$ lower phase noise than the two independent single-band oscillators. Phase-noise reduction by coupling multiple oscillators has been recognized in the RF and microwave circuit design community [16]-[18]. The phase noise was proved in theory to scale down as $1 / N$ if $N$ oscillators are ideally coupled [16]. However, coupling is far from trivial in real circuit design, and various structures were proposed [16]-[18]. Along this line, the proposed dual-band oscillator demonstrates the first ideally coupled structure with band switching capability. In a sense, the two inductors, which never idle, are better utilized to enhance phase noise than in the two-oscillator scheme.

Moreover, the proposed dual-band structure provides an alternative way of trading power for phase noise, and can achieve low phase noise that is impossible for a single-tank $L C$ oscillator (Fig. 2). For a single-tank $L C$ oscillator, this power versus phasenoise tradeoff is often done by halving the tank impedance and doubling the power consumption, which lowers the phase noise by half [18], but in low-supply-voltage applications, this conventional way may lead to impractically small inductance to meet the stringent phase-noise specifications [18]. In this case, the proposed structure can be used to reduce the phase noise by another $3 \mathrm{dBc} / \mathrm{Hz}$ without further scaling the inductance to impractical values. Besides, its band-switching capacity eases the tradeoff between phase noise and frequency tuning.

In the remainder of this paper, we will start with the operation of the proposed oscillator in Section II. In Section III, we will discuss its phase-noise performance. In Section IV, we will verify our analysis by a prototype and present measurement results. In closing, we will present a conclusion in Section V.

\section{OPERATION OF THE DuAL-MODE LC OSCILLATOR}

As illustrated in Fig. 3, the proposed oscillator consists of two parts: an $L C$ resonator and a trans-conductance network. Denoted by $Z(s)$ and $G$, respectively, these two parts form a feedback loop. We will study the oscillation condition of the oscillator using this feedback network model.

\section{A. LC Resonator}

Since we use center-taped symmetric inductors and crossconnected differential pairs as an active core, as shown in Fig. 1, the oscillator only works differentially. That is, the voltages at the two terminals of each inductor are opposite in sign. Thus, we are only interested in the resonator's differential modes. Analysis shows it has two resonant modes: an even mode and an odd mode. Instead of an abstract mathematical derivation, we introduce the two modes in an intuitive way.

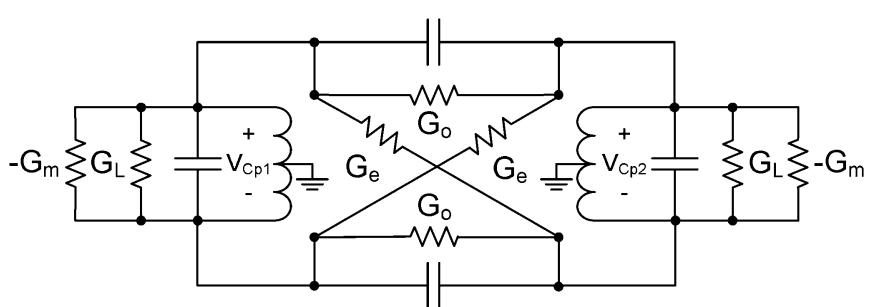

(a)

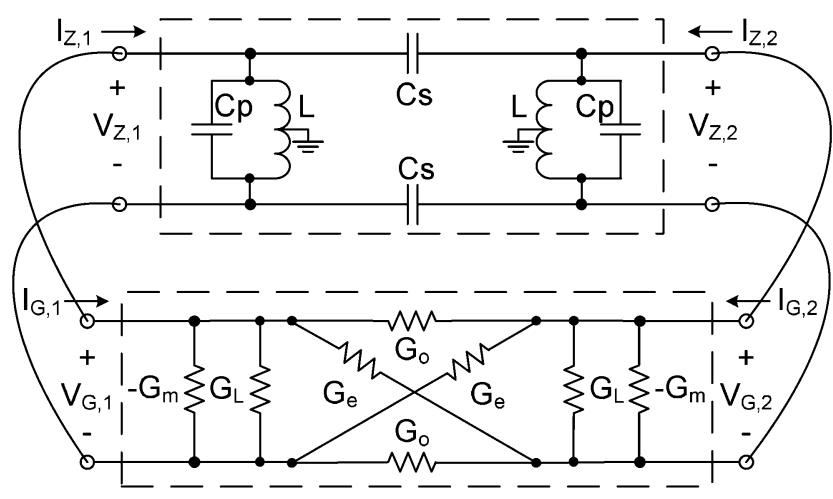

(b)

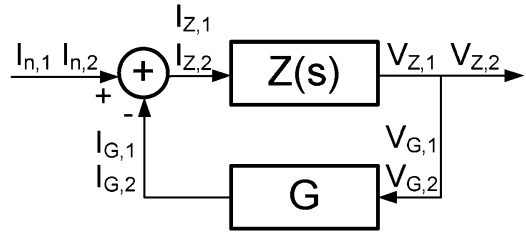

(c)

Fig. 3. Dual-mode $L C$ oscillator. (a) Physical model. (b) Redrawn physical model as two-port networks, i.e., an $L C$ resonator and a trans-conductance network. (c) Mathematical model.

1) In the even mode, the two $L C$ tanks resonate in phase, i.e., $V_{C p 1}=V_{C p 2}$. As illustrated in Fig. 4, the capacitors $C_{s}$ see a zero voltage drop and do not carry current. Thereby, the resonator can be reduced to two $L C$ tanks. The resonant frequency is easily found to be

$$
\omega_{e}=\frac{1}{\sqrt{L C_{p}}}
$$

in which the subscript $e$ stands for "even mode."

2) In the odd mode, the two $L C$ tanks are $180^{\circ}$ out of phase, i.e., $V_{C p 1}=-V_{C p 2}$. As illustrated in Fig. 5, the capacitor $C_{s}$ sees differential voltage at its two terminals. Thereby, it is virtual ground at the center of $C_{s}$. Breaking $C_{s}$ up in the middle, we get the equivalent circuit on the right side of Fig. 5, which consists of two $L C$ tanks. Thus, the resonant frequency is easily found to be

$$
\omega_{o}=\frac{1}{\sqrt{L\left(C_{s}+C_{p}\right)}}
$$

in which the subscript $o$ stands for "odd mode."

A complete description of the resonator is to model it as a two-port network, as shown in Fig. 3(b), and use its impedance matrix ( $Z$ matrix) [19], i.e.,

$$
\left[\begin{array}{l}
V_{Z, 1}(s) \\
V_{Z, 2}(s)
\end{array}\right]=Z(s) \cdot\left[\begin{array}{l}
I_{Z, 1}(s) \\
I_{Z, 2}(s)
\end{array}\right]
$$



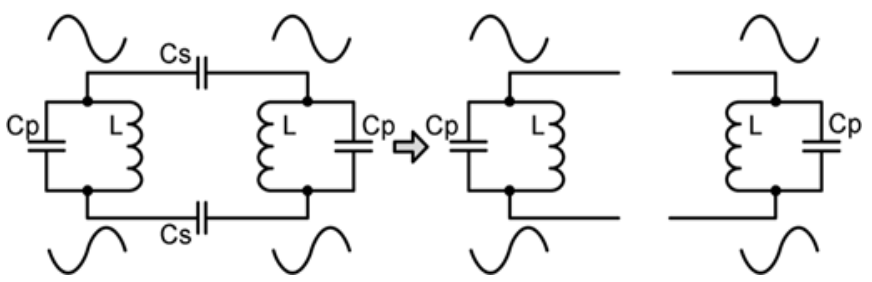

Fig. 4. Illustration of the even mode of the $L C$ resonator.

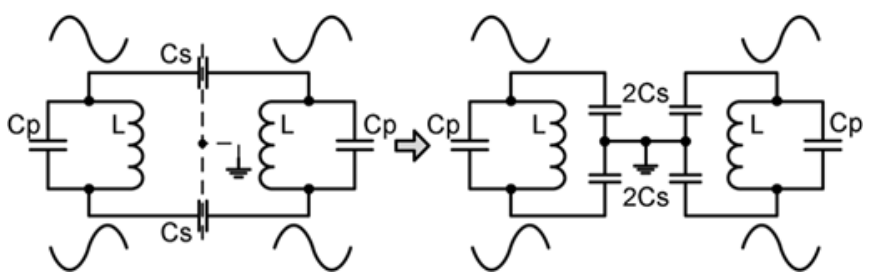

Fig. 5. Illustration of the odd mode of the $L C$ resonator.

and (5), shown at the bottom of this page. The two resonant frequencies are clearly shown by the two terms of each matrix element. For instance, the impedance looking into Port1 when Port2 is open, i.e., $\left|Z_{11}(j \omega)\right|$, and has two peaks, at $\omega_{e}$ and $\omega_{o}$, respectively. As an illustration, we implemented the resonator in a CMOS process. The impedance looking into Port1 is plotted in Figs. 6 and 7. As expected, there are two peaks in each curve, corresponding to the odd and even mode, respectively. The frequencies of the peaks are as predicted by (2) and (3). In Fig. 6, when $C_{s}$ increases, the odd-mode frequency $\omega_{o}$ decreases, whereas the even-mode frequency $\omega_{e}$ is not affected; in Fig. 7, when $C_{p}$ increases, both $\omega_{o}$ and $\omega_{e}$ decrease. It is worth mentioning that the two frequencies are not harmonically related, and their ratio $\omega_{e} / \omega_{o}=\sqrt{1+C_{s} / C_{p}}$ is determined by $C_{s} / C_{p}$.

\section{B. Trans-Conductance Network}

As illustrated in Fig. 3, we use a trans-conductance network to model all energy-loss and energy-compensation components in the oscillator. In particular, as in Fig. 3(a):

- $G_{L}$ is the parallel conductance of the resonator, and models the energy loss of the passive components;

- $-G_{m}$ is the negative conductance of the differential pairs;

- $G_{O}$ is the conductance of switches $S_{1}$ and $S_{2}$ in Fig. 1;

- $G_{e}$ is the conductance of switches $S_{3}$ and $S_{4}$ in Fig. 1.

Note that, when a switch turns off, its conductance $G_{o}$ or $G_{e}$ is zero; when it turns on, $G_{o}$ or $G_{e}$ is a positive value $G_{o \text {,on }}$

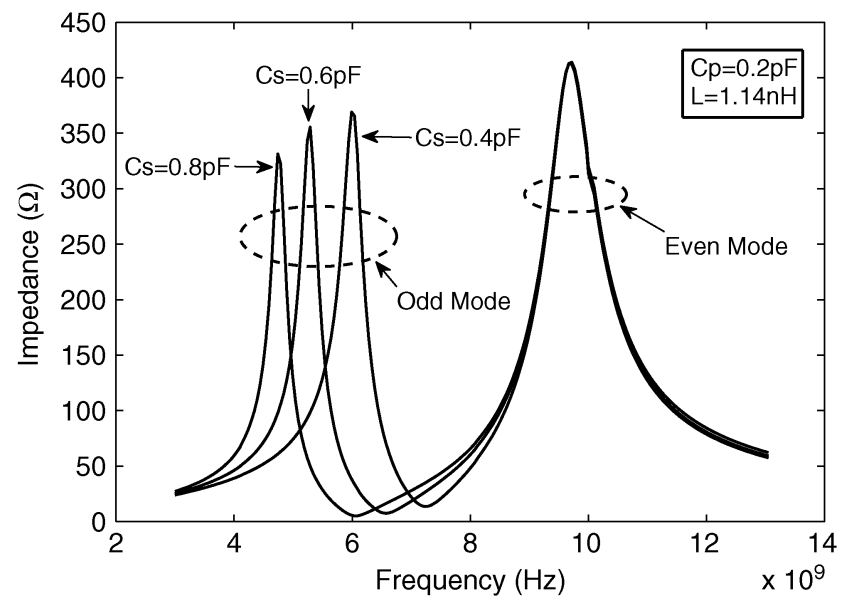

Fig. 6. Input impedance of the dual-band $L C$ resonator, with $C_{s}$ being tuned.

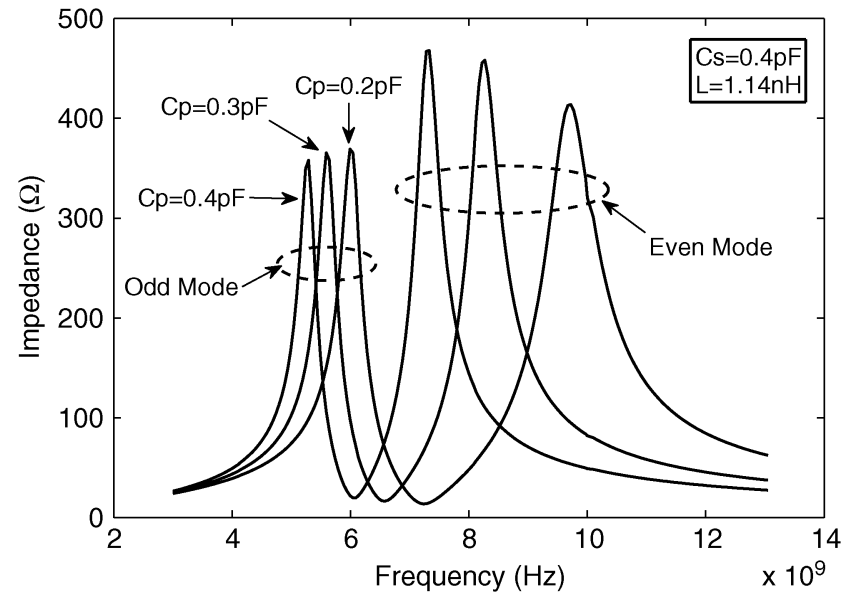

Fig. 7. Input impedance of the dual-band $L C$ resonator, with $C_{p}$ being tuned.

or $G_{e, \text { on }}$. Putting everything together, we obtain the two-port network shown in Fig. 3(b), which is described by

$$
\left[\begin{array}{c}
I_{G, 1} \\
I_{G, 2}
\end{array}\right]=G \cdot\left[\begin{array}{l}
V_{G, 1} \\
V_{G, 2}
\end{array}\right]
$$

and (7), shown at bottom of the following page. Interestingly, this network also shows even/odd operations. That is, if applying even voltage to the two ports, i.e., $V_{G, 1}=V_{G, 2}=V_{0}$, we get $I_{G, 1}=I_{G, 2}=\left(-G_{m}+G_{L}+G_{e}\right) \cdot V_{0}$. Thus, each port sees an effective conductance of $\left(-G_{m}+G_{L}+G_{e}\right)$; if applying odd voltage $V_{G, 1}=-V_{G, 2}$, each port sees an effective conductance of $\left(-G_{m}+G_{L}+G_{o}\right)$. As a result, even and odd modes of the $L C$ resonator see different energy loss and compensation, which

$$
Z(s)=\frac{1}{2}\left[\begin{array}{ll}
\frac{s}{C_{p}\left(s^{2}+\omega_{e}^{2}\right)}+\frac{s}{\left(C_{p}+C_{s}\right)\left(s^{2}+\omega_{o}^{2}\right)} & \frac{s}{C_{p}\left(s^{2}+\omega_{e}^{2}\right)}-\frac{s}{\left(C_{p}+C_{s}\right)\left(s^{2}+\omega_{o}^{2}\right)} \\
\frac{s}{C_{p}\left(s^{2}+\omega_{e}^{2}\right)}-\frac{s}{\left(C_{p}+C_{s}\right)\left(s^{2}+\omega_{o}^{2}\right)} & \frac{s}{C_{p}\left(s^{2}+\omega_{e}^{2}\right)}+\frac{s}{\left(C_{p}+C_{s}\right)\left(s^{2}+\omega_{o}^{2}\right)}
\end{array}\right]
$$


we can use to realize mode/frequency switching. Rigorous analysis is given in Section II-C.

\section{Feedback Network}

Based on above discussion and the mathematical model in Fig. 3(c), we can derive the response of the oscillator to noise current $\left[I_{n, 1}, I_{n, 2}\right]$ to be

$$
\begin{aligned}
{\left[\begin{array}{l}
V_{Z, 1}(s) \\
V_{Z, 2}(s)
\end{array}\right]=} & Z(s)[I+Z(s) G]^{-1} \cdot\left[\begin{array}{c}
I_{n, 1}(s) \\
I_{n, 2}(s)
\end{array}\right] \\
= & H_{e}(s) \frac{I_{n, 1}(s)+I_{n, 2}(s)}{2}\left[\begin{array}{l}
1 \\
1
\end{array}\right] \\
& +H_{o}(s) \frac{I_{n, 1}(s)-I_{n, 2}(s)}{2}\left[\begin{array}{c}
1 \\
-1
\end{array}\right]
\end{aligned}
$$

in which

$$
\begin{aligned}
& H_{e}(s)=\frac{1}{C_{p}} \cdot \frac{s}{s^{2}-\frac{G_{m}-G_{L}-G_{e}}{C_{p}} s+\omega_{e}^{2}} \\
& H_{o}(s)=\frac{1}{C_{p}+C_{s}} \cdot \frac{s}{s^{2}-\frac{G_{m}-G_{L}-G_{o}}{C_{p}+C_{s}} s+\omega_{o}^{2}} .
\end{aligned}
$$

From (8), we find even-mode noise can stimulate oscillation at $\omega_{e}$ with transfer function $H_{e}(s)$ and odd-mode noise can stimulate oscillation at $\omega_{o}$ with transfer function $H_{o}(s)$. Since random noise has both even- and odd-mode components, whether a mode can start up is determined by its transfer function $H_{e}(s)$ or $H_{o}(s)$.

- In even mode oscillation, switches $S_{1}$ and $S_{2}$ are on, and $S_{3}$ and $S_{4}$ are off. Thus, $G_{e}=0$ and $G_{o}=G_{o, \text { on }}>0$. Thereby, by sizing transistors such that

$$
G_{o, \text { on }}>G_{m}-G_{L}>0
$$

we can make $G_{m}-G_{L}-G_{e}>0$ and $G_{m}-G_{L}-G_{o}<0$. In this case, the even-mode transfer function $H_{e}(s)$ has its poles $p_{e}$ on the right-half plane, while the odd-mode transfer function $H_{o}(s)$ has its poles $p_{o}$ on the left-half plane. Therefore, only the even mode can start up.

- In odd-mode oscillation, switches $S_{1}$ and $S_{2}$ are off, and $S_{3}$ and $S_{4}$ are on. Thus, $G_{e}=G_{e, \text { on }}>0$ and $G_{o}=0$. Thereby, if the transistors are sized such that

$$
G_{e, \text { on }}>G_{m}-G_{L}>0
$$

we can make $G_{m}-G_{L}-G_{e}<0$ and $G_{m}-G_{L}-G_{o}>0$. In this case, $H_{e}(s)$ has its poles on the left-half plane, while
$H_{o}(s)$ has its poles on the right-half plane. Therefore, only the odd mode can start up.

To sum up, the transistors should be sized such that

$$
G_{e, \text { on }}, G_{o, \text { on }}>G_{m}-G_{L}>0
$$

in order to enable frequency switching, i.e., to guarantee startup of either oscillation mode and damp the other mode.

It is worth noting that $G_{e}$ and $G_{o}$ only damp the unwanted mode and do not degrade the working mode quality factor. Take the odd-mode oscillation as an example, in which $G_{e}=G_{e, \text { on }}>0$ and $G_{o}=0$. We simulated the input impedance of the resonator, with $G_{m}=G_{o}=0$ and $G_{e}$ changing from 0 to $0.004 \Omega^{-1}$. As illustrated in Fig. 8, $G_{e}$ lowers the even-mode peak, but does not affect the height or width of the odd-mode peak. Intuitively, the even-mode component imposes a voltage drop across $G_{e}$ and thus its energy is dissipated, but the odd-mode component does not see $G_{e}$ and is not affected. Similarly, in even-mode oscillation, $G_{o}=G_{o, \text { on }}>0$ only damps the odd mode and does not affect the even mode, as illustrated in Fig. 9. Thereby, we can expect good phase-noise performance in the dual-band oscillator. A rigorous analysis of phase noise will be given in Section III.

\section{Continuous Frequency Tuning}

In a single-band $L C \mathrm{VCO}$, people use a switched capacitor bank for coarse tune and a varactor for fine tune. However, due to the tradeoff between switch loss and parasitic capacitance, $C_{\max } / C_{\min }$ cannot be very large. For instance, [5] demonstrates a state-of-the-art design with a continuous tuning range of 3.1-5.2 GHz. This corresponds to $C_{\max } / C_{\min }=2.8$, which includes parasitics from active core and loading, etc.

The proposed scheme in Fig. 1 can be used to extend above tuning range. For instance, assume $C_{p}$ is implemented as switched capacitor and varactor with a tuning range between $C_{\max }$ and $C_{\min },{ }^{1}$ and $C_{s}$ is the fixed capacitor. Thus, the odd mode covers a low band from $1 / \sqrt{L\left(C_{\max }+C_{s}\right)}$ to $1 / \sqrt{L\left(C_{\min }+C_{s}\right)}$, and the even mode covers a high band from $1 / \sqrt{L C_{\max }}$ to $1 / \sqrt{L C_{\min }}$. Since the switches in this design do not affect the working mode quality factor, they do not need to be extremely wide transistors and thus do not introduce significant parasitic capacitance. Thus, $C_{\max } / C_{\min }$ can achieve roughly the same value as the single-band VCO above. If $C_{\max }=2.6 C_{0}, C_{\min }=C_{0}$, and $C_{s}=1.5 C_{0}$, the even and odd bands have a considerable overlap and cover a continuous tuning range with $f_{\max } / f_{\min }>2$. In contrast, in conventional single-band $\mathrm{VCO}$, such a tuning range requires

\footnotetext{
${ }^{1}$ Parasitic capacitances of active core and loading are included.
}

$$
G=\left[\begin{array}{cc}
-G_{m}+G_{L}+\frac{1}{2} G_{o}+\frac{1}{2} G_{e} & -\frac{1}{2} G_{o}+\frac{1}{2} G_{e} \\
-\frac{1}{2} G_{o}+\frac{1}{2} G_{e} & -G_{m}+G_{L}+\frac{1}{2} G_{o}+\frac{1}{2} G_{e}
\end{array}\right]
$$




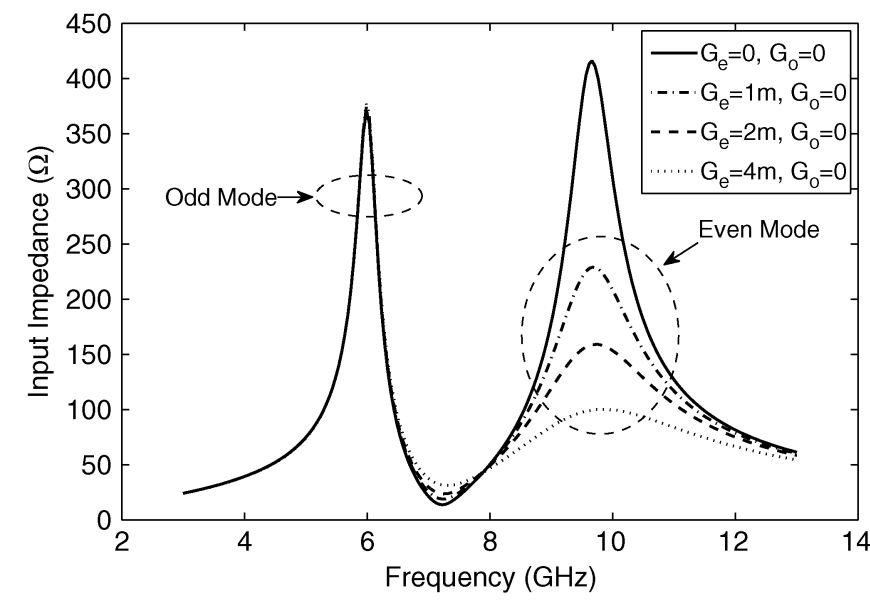

Fig. 8. Input impedance of dual-band resonator when $G_{e}$ changes.

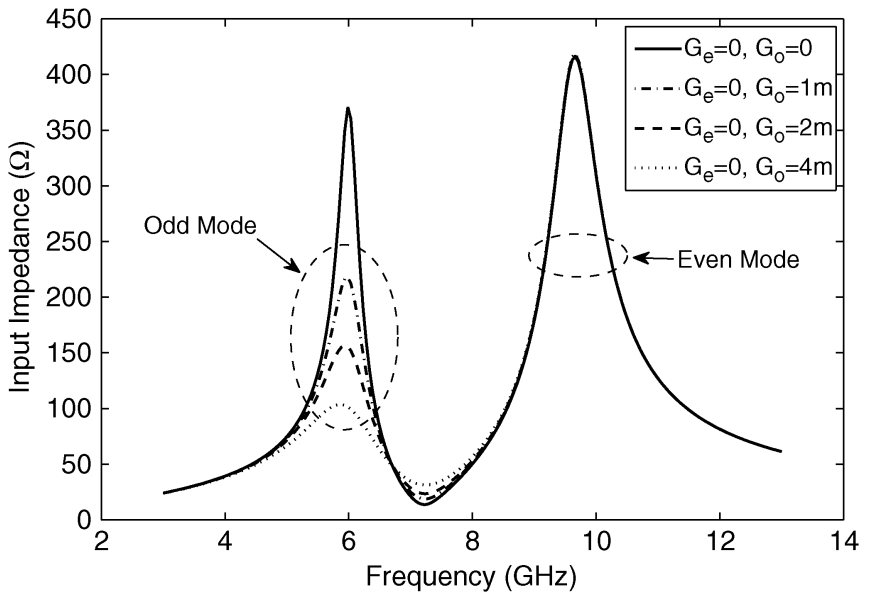

Fig. 9. Input impedance of dual-band resonator when $G_{0}$ changes.

$C_{\max } / C_{\min }>4$, which is hardily possible. In an implementation with 8-bit coarse tune bank and varactors in a $65-\mathrm{nm}$ CMOS technology, simulation shows the odd mode covers 2.4-3.2 GHz and the even band covers $3-5 \mathrm{GHz}$.

\section{Discussion ON PHASE NOISE}

Phase noise is a primary concern in oscillator design. Since the proposed dual-band structure can be considered as two coupled single-band oscillators, many analyses and phase-noise reduction techniques proposed for single-band oscillators (e.g., [13], [14], [22]) can be readily adopted here. Thereby, we will focus on the comparison between single- and dual-band oscillator, and demonstrate a $3-\mathrm{dBc} / \mathrm{Hz}$ phase-noise improvement. Existing phase-noise theories mainly fall into two categories [23]: frequency- [24] and time-domain techniques [15], [25]. Our analysis is based on the impulse sensitivity function (ISF) theory in [15].

\section{A. ISF}

We begin with the single-band $L C$ oscillator shown in Fig. 2. If a noise current pulse $i_{n}=\delta(t-\tau)$ is injected into the $L C$ tank at $t=\tau$, there would be a voltage jump of $\Delta V_{s}=1 / C$. This voltage jump induces a phase shift $\Delta \phi(\tau)$, which depends on the
$L C$ tank's state at $t=\tau$ and thus varies at the same frequency as the oscillator. $\Delta \phi(\tau)$ is actually the ISF function, describing the time-variant phase response to noise [15]. For oscillators with high- $Q L C$ resonators, the ISF can be well approximated by a sinusoidal function at the same frequency as the oscillator [20]. Assuming the oscillation swing is $V_{p}$, one can find the amplitude of the ISF is

$$
|\mathrm{ISF}|_{\text {single }}=\left|\frac{\Delta V_{s}}{V_{p}}\right|=\frac{1}{C \cdot V_{p}} .
$$

Next, we will consider the dual-band oscillator in Fig. 1. Take odd-mode oscillation as an example. Based on (8) and (10), if a noise current pulse is injected from $I_{n, 1}$ (or $I_{n, 2}$ ), the induced odd-mode voltage jump is $\Delta V_{o}=1 / 2\left(C_{p}+C_{s}\right)$. Although the current pulse also induces an even-mode component, it decays rapidly and does not change the phase of odd-mode oscillation in a first-order estimate. Thus, assuming the oscillation swing is $V_{p}$ and by the same reasoning as the single-band oscillator, one can find the amplitude of ISF is

$$
|\mathrm{ISF}|_{\text {odd }}=\left|\frac{\Delta V_{o}}{V_{p}}\right|=\frac{1}{2\left(C_{p}+C_{s}\right) \cdot V_{p}} .
$$

If the two oscillators use the same $L$ and are at the same frequency, we must have $C=C_{s}+C_{p}$. If also using the same active core and supply voltage, they should have the same voltage swing $V_{p}$. Therefore, from (14) and (15), we find

$$
|\mathrm{ISF}|_{\text {odd }}=\frac{1}{2}|\mathrm{ISF}|_{\text {single }}
$$

Intuitively, this result can be explained by the fact that, in the dual-mode oscillator, only half of the injected current pulse induces odd-mode voltage and thus shifts oscillation phase, while the other half induces even-mode component, which is damped by the circuit and does not perturb oscillation phase.

To verify this result, we implemented the single- and dualband $L C$ oscillator in a $0.13-\mu \mathrm{m}$ CMOS process and did simulation with SpectreRF. The two oscillators use the same inductor and active core, and the capacitors are set such that they are at the same frequency $5.39 \mathrm{GHz}$. Fig. 10 shows the simulated voltage waveforms at a terminal of the inductors, along with the ISFs of a noise source taped to the same net. The voltage swings are the same for the two oscillators, and considering $V d d=0.5 \mathrm{~V}$, both work just in the voltage-limited region [21]. The ISFs are at the same frequency as the voltages, and the phase is shown to be most sensitive to noise at voltage zero-crossings [15]. We calculate the root-mean-square (rms) values as the amplitudes of the ISF curves. The ISF of the dual-band oscillator is exactly half of the amplitude of the single-band oscillator. The ISFs of noise sources at other nets also show the same ratio.

In the same way, we found the same comparison result for the dual-band oscillator's even mode, provided the single-band oscillator is set to the same frequency. For the sake of space, we will not repeat the discussion for the even mode here.

\section{B. Noise Sources}

In addition to the ISFs, noise sources are the other factor to determine phase-noise performance. Although the two oscil- 

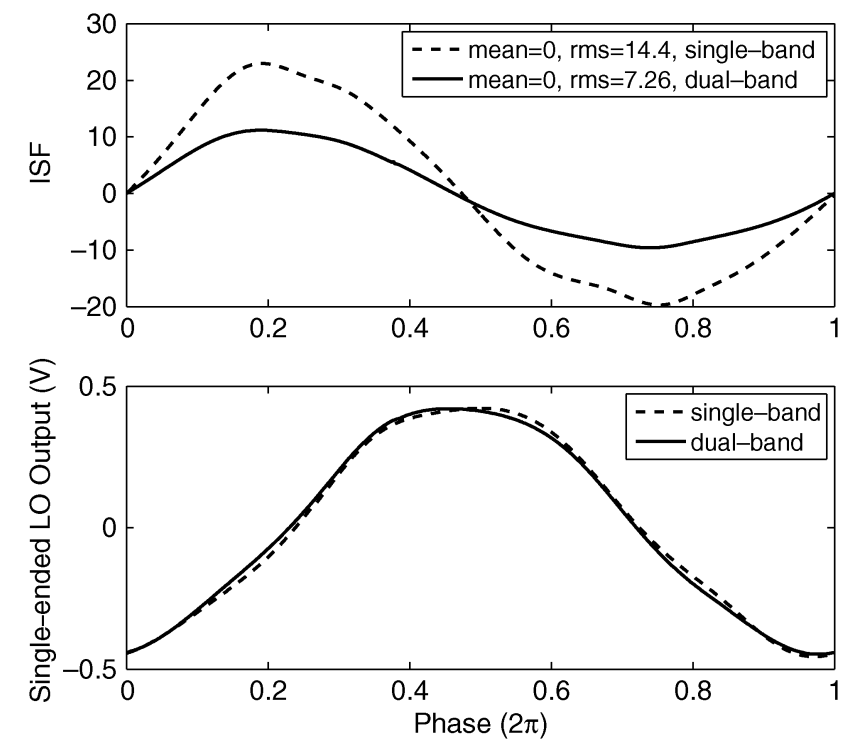

Fig. 10. Simulated ISFs and voltage waveforms of the single- and dual-band oscillator in the odd mode. The two subplots are aligned in time.
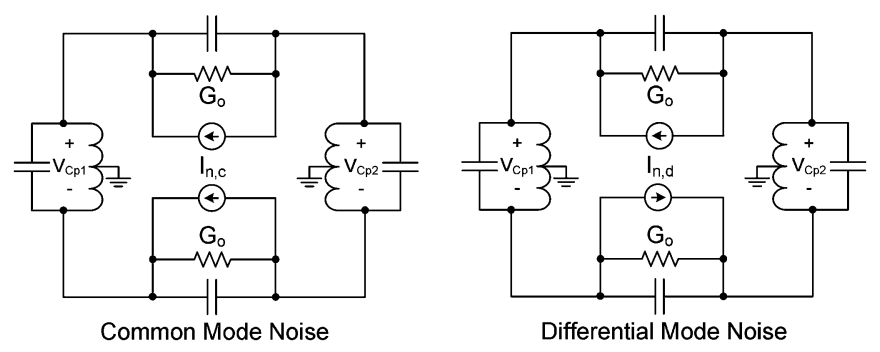

Fig. 11. Illustration of switch noise in even mode oscillation.

lators have common noise sources in active cores and inductors, the switches in the dual-band oscillator introduce extra noise sources and need careful checking. Take the even-mode oscillation as an example. Switches $S_{1}$ and $S_{2}$ are on in the even-mode oscillation (Fig. 1), and insert two nonzero conductors $G_{o}$, as shown in Fig. 11. The noise of the two $G_{o}$ 's can be modeled as two current sources, which can always be decomposed into its common- and differential-mode components: The common-mode noise is damped by the active cores and the center-tapped symmetric inductors in that they only support differential mode oscillation and the common mode component cannot sustain; the differential-mode noise leads to an odd-mode component in the resonator, which is damped by $G_{O}$ 's. In other words, in even-mode oscillation, although the two $G_{o}$ 's generate noise, they do not add any even-mode component to the resonator, and thus does not perturb the phase of oscillation. Along the same line, one can find the same result for the odd-mode oscillation. SpectreRF simulation verified this result. Table I lists itemized phase-noise contribution from different parts of the circuits. The switching network accounts for only $0.9 \%$ of the total noise and is thus negligible in phase-noise analysis.

\section{Phase Noise}

According to the ISF theory of phase noise [15], the phase noise induced by a noise source is proportional to the square of its ISF's amplitude. Based on the ISF comparison in (16), the
TABLE I

Simulated Phase-Noise Contribution at $\Delta f=1 \mathrm{MHz}$

\begin{tabular}{|c|c|c|}
\hline & Single-band & Dual-band \\
\hline active core & $69.6 \%$ & $66.9 \%$ \\
inductor & $22.3 \%$ & $22.7 \%$ \\
capacitor & $6.1 \%$ & $5.8 \%$ \\
switches & N/A & $0.9 \%$ \\
others & $2 \%$ & $3.7 \%$ \\
\hline
\end{tabular}

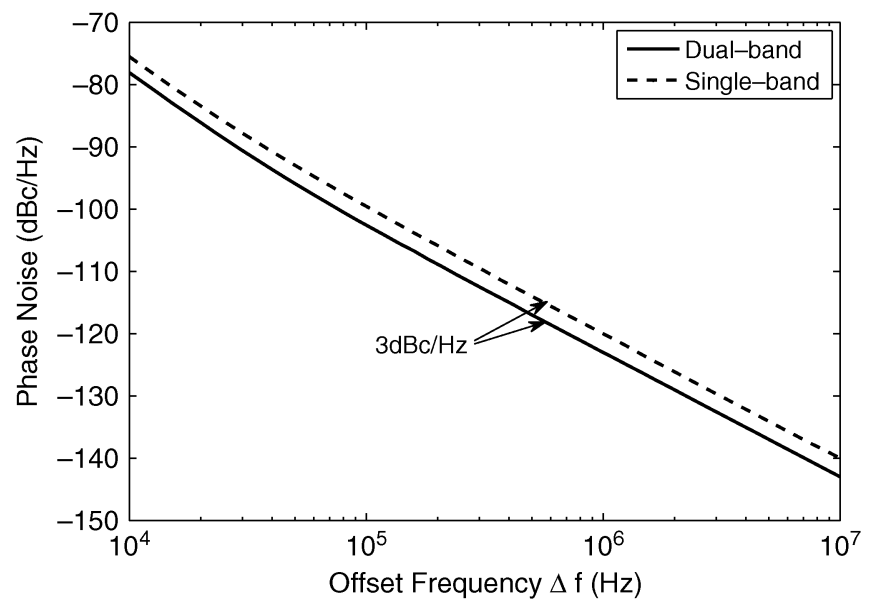

Fig. 12. Simulated phase noise of the single-band oscillator and the dual-band oscillator in the odd mode.

phase noise of the dual-band oscillator, due to one active core and/or one inductor, is $(1 / 2)^{2}=1 / 4$ of that of the single-band oscillator. On the other hand, the dual-band oscillator has two inductors and two active cores, which introduces twice the noise sources of the single-band oscillator. Therefore, the total phase noise of the dual-band oscillator is $2 \times(1 / 4)=1 / 2$ of the single-band one, which is a $3-\mathrm{dBc} / \mathrm{Hz}$ improvement. Moreover, because the dual-band oscillator has two active cores, it consumes twice the power of the single-band oscillator. Therefore, we conclude the dual- and single-band oscillators have the same FOM, as defined in (1).

This was verified by SpectreRF simulation. The single- and dual-band oscillators draw a current of 2 and $4 \mathrm{~mA}$, respectively, from a $0.5-\mathrm{V}$ power supply. We set the dual-band oscillator to be in odd mode, and set the capacitors of the single-band oscillator such that it is at the same frequency. The simulated phase noises are plotted in Fig. 12. As expected, the dual-band oscillator shows $3-\mathrm{dBc} / \mathrm{Hz}$ lower phase noise than the single-band oscillator. The same result also holds for a dual-band oscillator working in the even mode and a single-band oscillator at the same frequency.

\section{Comparison With Other LO Schemes}

At this point, we can compare the proposed oscillator with other single- and dual-band LO schemes.

Compared to a single-band $L C$ oscillator in Fig. 2, the dual-band oscillator can be considered as a coupling structure and provides an alternative way of trading power for phase noise. For a single-band $L C$ oscillator in Fig. 2, the conventional way of reducing phase noise by half is to halve the $L C$ tank's impedance and double power consumption [18]. However, this method may lead to inductance values too small to be practical, 
especially when the phase-noise requirement is stringent [18]. In this case, coupling multiple $L C$ oscillators provides an alternative way of trading power for phase noise [16]-[18]. It is proven in theory, for $N$ ideally coupled oscillators, the phase noise reduces to $1 / N$ while the power increases to $N$ times of a single oscillator [16]. Along this line, the proposed oscillator can be recognized as an ideally coupled structure, which can lower phase noise by another $3 \mathrm{dBc} / \mathrm{Hz}$ without scaling down inductances to impractical values. Although this $3-\mathrm{dBc} / \mathrm{Hz}$ benefit can also be achieved by directly connecting two $L C$ tanks in parallel, the proposed dual-band structure provides an additional band-switching capability, which eases the tradeoff between frequency tuning range and phase noise.

Compared to a dual-band scheme with two independent oscillators [1], the dual-band oscillator readily get a $3-\mathrm{dBc} / \mathrm{Hz}$ improvement in phase noise by coupling them together. If two independent $L C$ oscillators work alternatively, there is always one inductor in idle, which could have been used to improve phase noise of the working oscillator. In this sense, the proposed dual-band structure makes better use of the inductors, which never idle, to get $3-\mathrm{dBc} / \mathrm{Hz}$ lower phase noise, while keeping the capability of synthesizing two frequencies.

With respect to other dual-band structures, it is not easy to make a fair quantitative comparison directly. A single-band $L C$ oscillator should be a good reference here. As discussed above, the proposed dual-band oscillator can achieves the same phasenoise FoM as a single-band one, which is seldom possible for other designs [2]-[12]. Moreover, since the proposed dual-band structure can be considered as two coupled single-band $L C$ oscillators, many phase-noise reduction techniques proposed for single-band $L C$ oscillators [13], [14] can be readily adopted in the proposed dual-band design.

\section{VERIFICATION BY PROTOTYPES}

We implemented a dual-band (Fig. 1) and a single-band oscillator (Fig. 2) in a $0.13-\mu \mathrm{m}$ CMOS technology with $0.5-\mathrm{V}$ power supply. The two use the same PFET pair $(\mathrm{W} / \mathrm{L}=72 \mu \mathrm{m} / 0.12 \mu \mathrm{m})$ and the same center-taped symmetric inductor $(1.138 \mathrm{nH})$ built on the two thick metals. We use metal-insulator-metal (MIM) capacitors, the values of which are set such that the oscillators work at the desired frequencies.

\section{A. Switch Network}

In the prototype, the switch network was modified from Fig. 1 to 13 , mainly because the large voltage swing may turn switches on while they should remain off. Take $S_{1}$ as an example. In an odd-mode oscillation, it is turned off by setting its gate voltage to zero. However, the oscillation voltage at the inductor terminals can drop below $-420 \mathrm{mV}$, which can turn $S_{1}$ on shortly. This leads to a leaking current, which degrades the quality factor of the resonator. To solve this problem, we used a capacitive voltage divider in Fig. 13. In this way, switch transistors only see half of the voltage swing and the leaking current is eliminated. Meanwhile, the switch transistors $(\mathrm{W} / \mathrm{L}=40 \mu \mathrm{m} / 0.12 \mu \mathrm{m})$ are twice as wide as before to provide the same damping effect.

It is worth mentioning that the modified switch network is not an essential part of the structure in that there are other ways to

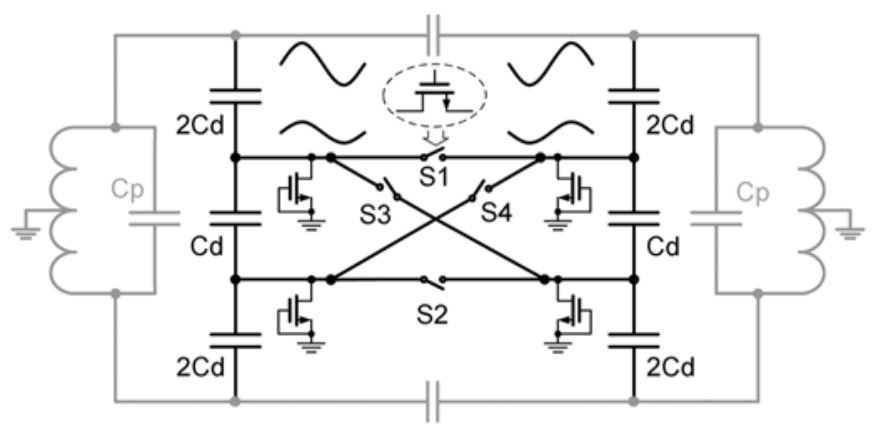

Fig. 13. Modified switching network to overcome current leakage.

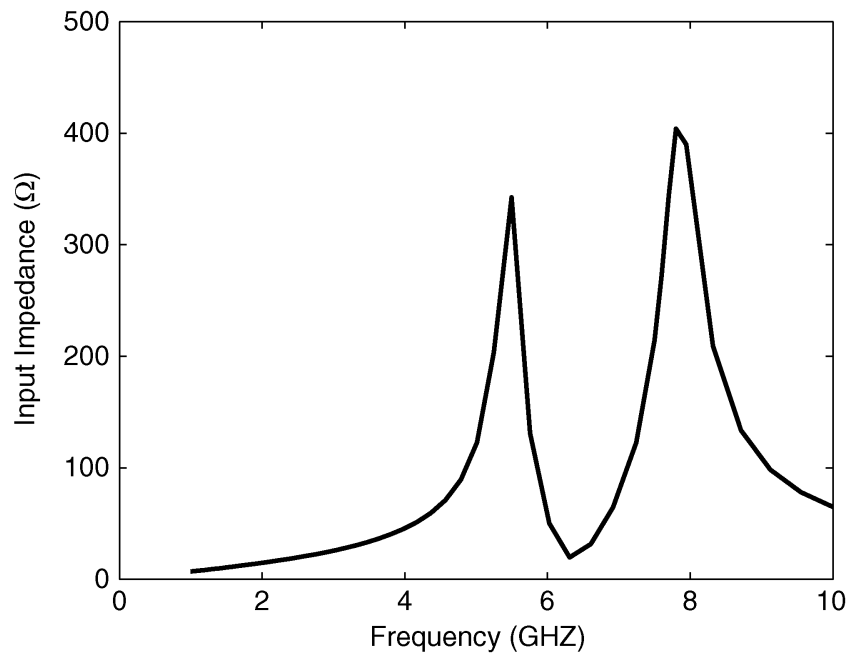

Fig. 14. Simulated input impedance of implemented resonator, loaded by active core. Two peaks appear at 5.4 and $7.7 \mathrm{GHz}$, corresponding to odd- and even-mode resonance, respectively.

avoid leakage current, e.g., CMOS instead of pMOS active core. We will not go to details for the sake of space.

\section{B. Fabrication and Measurement Result}

A single- and dual-band oscillator were fabricated in a $0.13-\mu \mathrm{m}$ CMOS process with the frequency of the single-band oscillator close to the odd mode of the dual-band oscillator. Since our purpose here is to verify the functionality of switching and analysis on phase noise, we did not include varactors for continuous tuning in this prototype design. Fig. 14 shows simulated input impedance of the implemented dual-mode $L C$ resonator, loaded with active core and switch network. Fig. 15 shows a die photograph of the dual-band oscillator. Both oscillators were measured with an Agilent 8564EC spectrum analyzer. The measured phase noises are in Figs. 16-18.

Tables II and III summarize and compare results from simulation and measurement. We find that, although the measured frequencies of both oscillators drop due to parasitics, the measured phase noise agrees well with simulation. Besides, the frequency of the dual-band oscillator drops more than that of the single band, which can be explained by the parasitics of the long metal traces between the two inductors.

In terms of phase noise, the single-band oscillator is at about the same frequency as the odd mode of the dual-band oscillator. As expected, both simulation and measurement show the 


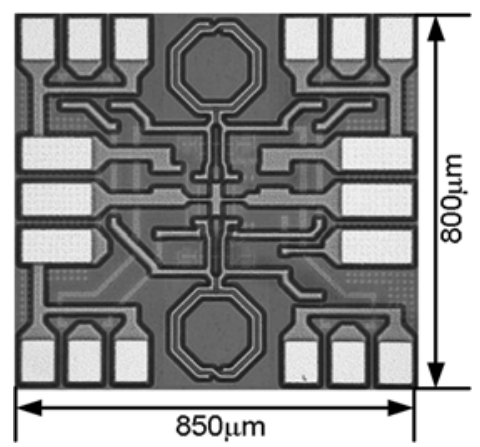

Fig. 15. Die photograph of the proposed dual-band oscillator.

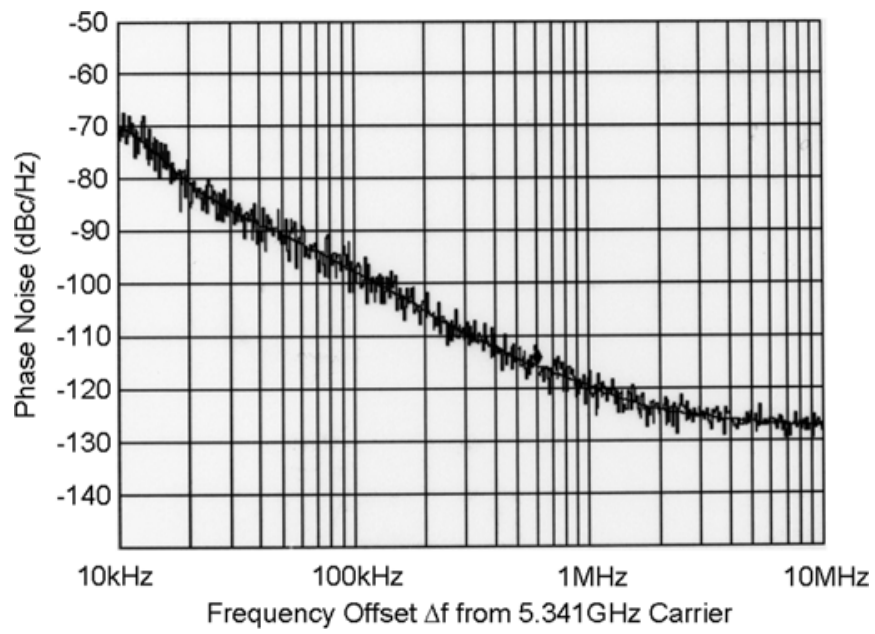

Fig. 16. Measured phase noise of a single-band oscillator: $L(\Delta f)=$ $-98.3 \mathrm{dBc} @ 100 \mathrm{kHz}$ and $-115.8 \mathrm{dBc} @ 600 \mathrm{kHz}$ with $f_{0}=5.341 \mathrm{GHz}$, $I_{\mathrm{DC}}=2.01 \mathrm{~mA}$, and $V_{D D}=0.504 \mathrm{~V}$.

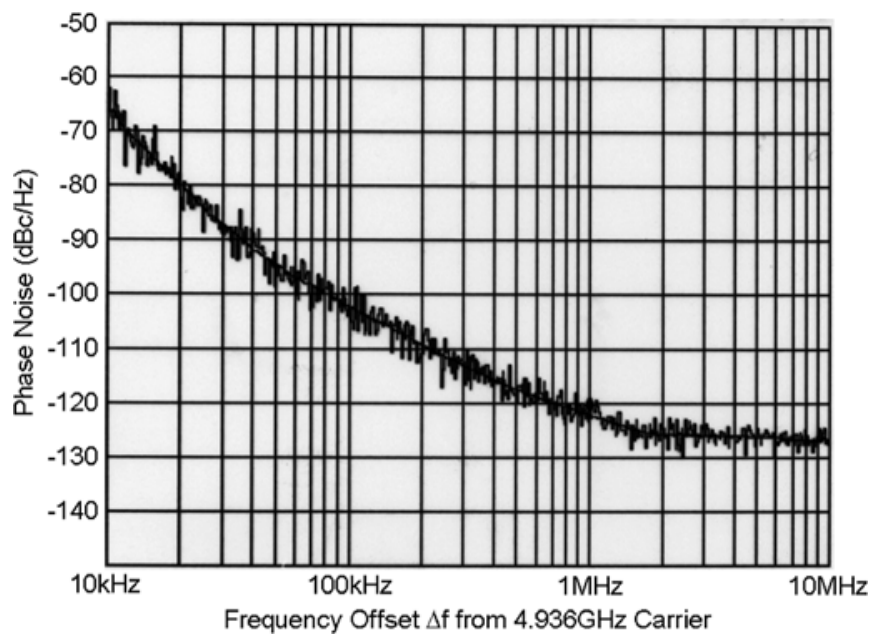

Fig. 17. Measured phase noise of the odd mode of a dual-band oscillator: $L(\Delta f)=-102.3 \mathrm{dBc} @ 100 \mathrm{kHz}$ and $-119.3 \mathrm{dBc} @ 600 \mathrm{kHz}$ with $f_{0}=4.936 \mathrm{GHz}, I_{\mathrm{DC}}=4.00 \mathrm{~mA}$, and $V_{D D}=0.506 \mathrm{~V}$.

3-dBc/Hz phase-noise improvement of the dual-band oscillator, compared to the single-band oscillator. Besides, the dual-band oscillator consumes twice the power of the single-band one. Thus, the two oscillators have the same phase-noise FoM, which verifies our analysis in Section III.

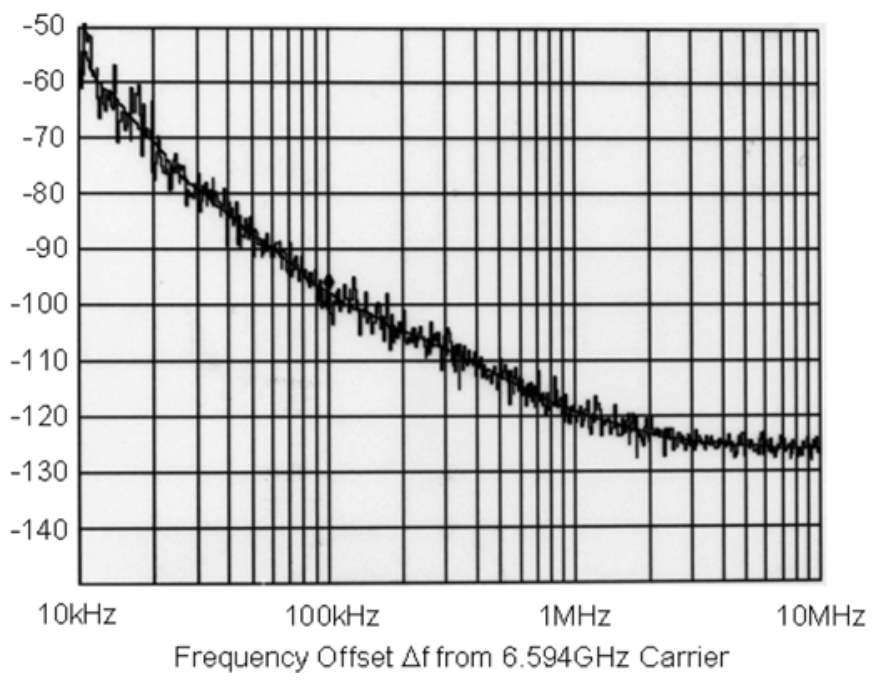

Fig. 18. Measured phase noise of the even mode of a dual-band oscillator: $L(\Delta f)=-97.7 \mathrm{dBc} @ 100 \mathrm{kHz}$ and $-114.8 \mathrm{dBc} @ 600 \mathrm{kHz}$ with $f_{0}=6.594 \mathrm{GHz}, I_{\mathrm{DC}}=3.99 \mathrm{~mA}$, and $V_{D D}=0.510 \mathrm{~V}$.

TABLE II

SUMMARY OF SIMULATION RESULTS

\begin{tabular}{|c|c|c|c|}
\hline & Single-band & $\begin{array}{c}\text { Dual-band } \\
\text { Odd Mode }\end{array}$ & $\begin{array}{c}\text { Dual-band } \\
\text { Even Mode }\end{array}$ \\
\hline$V_{D D}(\mathrm{~V})$ & 0.50 & 0.50 & 0.52 \\
\hline$I_{D C}(\mathrm{~mA})$ & 1.97 & 3.98 & 4.05 \\
\hline$f_{0}(\mathrm{GHz})$ & 5.479 & 5.390 & 7.583 \\
\hline$L(\Delta f)$ & $-99.3 @ 0.1$ & $-102.5 @ 0.1$ & $-97.9 @ 0.1$ \\
$(\mathrm{dBc} / \mathrm{Hz}$ & $-115.3 @ 0.6$ & $-118.5 @ 0.6$ & $-114.0 @ 0.6$ \\
$@ \mathrm{MHz})$ & $194.1 @ 0.1$ & $194.1 @ 0.1$ & $192.3 @ 0.1$ \\
\hline $\mathrm{FOM}(\Delta f)$ & $194.6 @ 0.6$ & $194.6 @ 0.6$ & $193.0 @ 0.6$ \\
$(\mathrm{~dB} @ \mathrm{MHz})$ & & & \\
\hline
\end{tabular}

TABLE III

SUMMARY OF MEASUREMENT RESULTS

\begin{tabular}{|c|c|c|c|}
\hline & Single-band & $\begin{array}{c}\text { Dual-band } \\
\text { Odd Mode }\end{array}$ & $\begin{array}{c}\text { Dual-band } \\
\text { Even Mode }\end{array}$ \\
\hline$V_{D D}(\mathrm{~V})$ & 0.504 & 0.506 & 0.510 \\
\hline$I_{D C}(\mathrm{~mA})$ & 2.01 & 4.00 & 3.99 \\
\hline$f_{0}(\mathrm{GHz})$ & 5.341 & 4.936 & 6.594 \\
\hline$L(\Delta f)$ & $-98.3 @ 0.1$ & $-102.3 @ 0.1$ & $-97.7 @ 0.1$ \\
$(\mathrm{dBc} / \mathrm{Hz}$ & $-115.8 @ 0.6$ & $-119.3 @ 0.6$ & $-114.8 @ 0.6$ \\
$@ \mathrm{MHz})$ & $192.8 @ 0.1$ & $193.1 @ 0.1$ & $191.0 @ 0.1$ \\
\hline $\mathrm{FOM}(\Delta f)$ & $194.7 @ 0.6$ & $194.5 @ 0.6$ & $192.5 @ 0.6$ \\
$(\mathrm{~dB} @ \mathrm{MHz})$ & 194.6 \\
\hline
\end{tabular}

The even-mode oscillates at higher frequency and its FoM is about $2 \mathrm{~dB}$ lower than the odd mode, largely because of the drop in the resonator's $Q$.

\section{CONCLUSION}

In this paper, we presented a distributed dual-band oscillator suitable for low-phase-noise applications. In contrast to other switched-resonator designs, there is actually no current going through the switches, which leads to low phase noise. The design and analysis were verified by a prototype implemented in a $0.13-\mu \mathrm{m}$ CMOS process. There is good agreement among theory, simulation, and measurement results. 


\section{ACKNOWLEDGMENT}

The authors would like to thank the Metal Oxide Semiconductor Implementation Service (MOSIS) and Taiwan Semiconductor Manufacturing Company (TSMC) University Shuttle Program for chip fabrication, O. Momeni, R. K. Dokania, Y. Tousi, and W. Lee, all with Cornell University, Ithaca, NY, for helpful technical discussions, and their wives M. Azarmnia and S. Lang for their support.

\section{REFERENCES}

[1] A. Kral, A. Behbahani, and A. A. Abidi, "RF-CMOS oscillators with switched tuning," in Proc. IEEE Custom Integr. Circuits Conf., 1998, pp. 555-558.

[2] S.-M. Yim and K. K. O, "Demonstration of a switched resonator concept in a dual-band monolithic CMOS $L C$-tuned VCO," in Proc. IEEE Custom Integr. Circuits Conf., 2001, pp. 205-208.

[3] N. D. Dalt, E. Thaller, P. Gregorius, and L. Gazsi, "A compact tripleband low-jitter digital $L C$ PLL with programmable coil in $130 \mathrm{~nm}$ CMOS," IEEE J. Solid-State Circuits, vol. 40, no. 7, pp. 1482-1490, Jul. 2005.

[4] Z. Li and K. K. O, "A low-phase-noise and low-power multiband CMOS voltage-controlled oscillator," IEEE J. Solid-State Circuits, vol. 40, no. 6, pp. 1296-1302, Jun. 2005.

[5] D. Hauspie, E. Park, and J. Craninckx, "Wideband VCO with simultaneous switching of frequency band, active core, and varactor size," IEEE J. Solid-State Circuits, vol. 42, no. 7, pp. 1472-1480, Jul. 2007.

[6] N. T. Tchamov, S. S. Broussev, I. S. Uzunov, and K. K. Rantala, "Dualband $L C$ VCO architecture with a fourth-order resonator," IEEE Trans. Circuits Syst. II, Exp. Briefs, vol. 54, no. 3, pp. 277-281, Mar. 2007.

[7] B. Catli and M. M. Hella, "A 1.94 to $2.55 \mathrm{GHz}, 3.6$ to $4.77 \mathrm{GHz}$ tunable CMOS VCO based on double-tuned double-driven coupled resonators," IEEE J. Solid-State Circuits, vol. 44, no. 9, pp. 2463-2477, Sep. 2009.

[8] G. Cusmai, M. Repossi, G. Albasini, A. Mazzanti, and F. Svelto, "A magnetically tuned quadrature oscillator," IEEE J. Solid-State Circuits, vol. 42 , no. 12 , pp. $2870-2877$, Dec. 2007.

[9] A. Goel and H. Hashemi, "Frequency switching in dual-resonance oscillators," IEEE J. Solid-State Circuits, vol. 42, no. 3, pp. 571-582, Mar. 2007.

[10] J. Borremans, A. Bevilacqua, S. Bronckers, M. Dehan, M. Kuijk, P. Wambacq, and J. Craninckx, "A compact wideband front-end using a single-inductor dual-band VCO in $90 \mathrm{~nm}$ digital CMOS," IEEE J. Solid-State Circuits, vol. 43, no. 12, pp. 2693-2705, Dec. 2008.

[11] S.-W. Tam, H.-T. Yu, Y. Kim, E. Socher, M. C. F. Chang, and T. Itoh, "A dual band mm-wave CMOS oscillator with left-handed resonator," in Proc. IEEE Radio Freq. Integr. Circuits Symp., 2009, pp. 477-480.

[12] Z. Safarian and H. Hashemi, "Wideband multi-mode CMOS VCO design using coupled inductors," IEEE Trans. Circuits Syst. I, Reg. Papers, vol. 56, no. 8, pp. 1830-1843, Aug. 2009.

[13] P. Andreani and A. Fard, "More on the $1 / f^{2}$ phase noise performance of CMOS differential-pair LC tank oscillators," IEEE J. Solid-State Circuits, vol. 41, no. 12, pp. 2703-2712, Dec. 2006.

[14] A. Mazzanti and P. Andreani, "Class-C harminic CMOS VCO with a general result on phase noise," IEEE J. Solid-State Circuits, vol. 43, no. 12, pp. 2716-2729, Dec. 2008.

[15] A. Hajimiri and T. H. Lee, "A general theory of phase noise in electrical oscillators," IEEE J. Solid-State Circuits, vol. 33, no. 2, pp. 179-194, Feb. 1998.

[16] H.-C. Chang, X. Cao, U. K. Mishra, and R. A. York, "Phase noise in coupled oscillators: Theory and experiment," IEEE Trans. Microw. Theory Tech., vol. 45, no. 5, pp. 604-615, May 1997.

[17] H. Jacobsson, B. Hansson, H. Berg, and S. Georgovian, "Very low phase-noise fully-integrated coupled VCOs," in IEEE MTT-S Int. Microw. Symp. Dig., Jun. 2002, vol. 1, pp. 577-580.

[18] L. Romanò, A. Bonfanti, S. Levantino, C. Samori, and A. L. Lacaita, "5-GHz oscillator array with reduced flicker up-conversion in $0.13-\mu \mathrm{m}$ CMOS," IEEE J. Solid-State Circuits, vol. 41, no. 11, pp. 2457-2467, Nov. 2006.
[19] D. M. Pozar, Microwave Engineering, 3rd ed. New York: Wiley, 2005.

[20] P. Andreani and X. Wang, "On the phase-noise and phase error performances of multiphase Lc CMOS VCOs," IEEE J. Solid-State Circuits, vol. 39 , no. 11, pp. 1883-1893, Nov. 2004

[21] A. Hajimiri and T. H. Lee, "Design issue in CMOS differential $L C$ oscillators," IEEE J. Solid-State Circuits, vol. 34, no. 5, pp. 717-724, May 1999.

[22] J. J. Rael and A. A. Abidi, "Physical processes of phase noise in differential LC oscillators," in Proc. IEEE Custom Integr. Circuits Conf., May 2000, pp. 569-572.

[23] A. Suarez, S. Sancho, S. Ver Hoeye, and J. Portilla, "Analytical comparison between time- and frequency-domain techniques for phase noise analysis," IEEE Trans. Microw. Theory Tech., vol. 50, no. 10, pp. 2353-2361, Oct. 2002

[24] K. Kurokawa, "Noise in synchronized oscillators," IEEE Trans. Microw. Theory Tech., vol. 16, no. 4, pp. 234-240, Apr. 1968

[25] A. Demir, A. Mehrotra, and J. Roychowdhury, "Phase noise in oscillators: A unifying theory and numerical methods for characterization," IEEE Trans. Circuits Syst. I, Fund. Theory Appl., vol. 47, no. 5, pp. 655-674, May 2000.

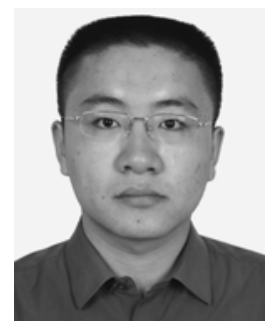

Guansheng Li (S'10) received the B.Sc. and M.Sc. degrees in electronic engineering from Tsinghua University, Beijing, China, in 2005 and 2007, respectively, and is currently working toward the $\mathrm{Ph} . \mathrm{D}$. degree in electrical and computer engineering at Cornell University, Ithaca, NY.

In 2010, he was an Intern with Qualcomm Inc., San Diego, CA, where he designed wide tuning-range voltage-control oscillators (VCOs) and frequency synthesizers. His current research interest is RF integrated circuit design with a focus on multiphase and multiband oscillator design and frequency synthesis. He has also conducted research on wireless communications and networking, studying cross-layer optimization and wireless network coding. He is a reviewer for Wireless Communications and Mobile Computing.

$\mathrm{Mr}$. $\mathrm{Li}$ is a reviewer for the IEEE TRANSACTIONS ON WIRELESS COMMUNICATIONS and the IEEE SENSORS JOURNAL. He was named a Jacobs Scholar at Cornell University in 2007.

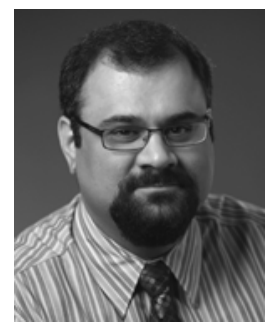

Ehsan Afshari (S'98-M'07) was born in 1979. He received the B.Sc. degree in electronics engineering from the Sharif University of Technology, Tehran, Iran, in 2001, and the M.S. and Ph.D. degrees in electrical engineering from the California Institute of Technology, Pasadena, in 2003, and 2006, respectively.

In August 2006, he joined the Faculty of Electrical and Computer Engineering, Cornell University, Ithaca, NY. His research interests are high-speed and low-noise integrated circuits for applications in communication systems, sensing, and biomedical devices.

Prof. Afshari was the chair of the IEEE Ithaca Section and chair of Cornell Highly Integrated Physical Systems (CHIPS). He is a member of the Analog Signal Processing Technical Committee, IEEE Circuits and Systems Society. He was the recipient of the National Science Foundation CAREER Award in 2010, Cornell College of Engineering Michael Tien Excellence in Teaching Award in 2010, Defense Advanced Research Projects Agency Young Faculty Award in 2008, and Iran's Best Engineering Student Award presented by the President of Iran in 2001. He was also the recipient of the Best Paper Award of the Custom Integrated Circuits Conference (CICC) in 2003, First Place of the Stanford-Berkeley-Caltech Inventors Challenge in 2005, the Best Undergraduate Paper Award of the Iranian Conference on Electrical Engineering in 1999, the Silver Medal of the Physics Olympiad in 1997, and the Award of Excellence in Engineering Education of the Association of Professors and Scholars of Iranian Heritage (APSIH) in 2004. 